\title{
Autotransplante de cordas tendíneas: nova técnica para o tratamento cirúrgico da insuficiência mitral por rotura de cordas tendíneas da cúspide anterior
}

Francisco GREGORI Jr. *, S. S. SILVA *, Luciano A. FAÇANHA **, Osney MOURE **, Marcos P.

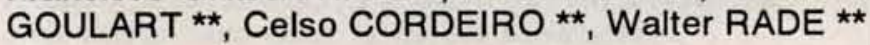

RBCCV 44205-186

GREGORI Jr., F.; SILVA, S. S.; FAÇANHA, L. A.; MOURE, O.; GOULART, M. P.; CORDEIRO, C.; RADE, W. - Autotransplante de cordas tendíneas: nova técnica para o tratamento cirúrgico da insuficiência mitral por rotura de cordas tendíneas da cúspide anterior. Rev. Bras. Cir. Cardiovasc., 7(4):250-255, 1992.

RESUMO: Uma das causas mais freqüentes de prolapso valvar mitral, com conseqüente regurgitaçāo, é a rotura de cordas tendíneas. Várias técnicas têm sido empregadas em tal situaçāo. Entretanto, a substituiçảo valvar mitral tem sido o procedimento mais utilizado. É apresentada uma nova técnica cirúrgica empregada em 4 pacientes com insuficiência valvar mitral severa devido a rotura de cordas tendíneas da cúspide anterior da valva mitral. Um autotransplante de cordas da valva tricúspide para a valva mitral é realizado. Em 2 casos, um retalho é retirado da cúspide anterior da valva tricúspide com cordas tendíneas e parte do músculo papilar correspondente. Nos outros 2 casos a cúspide posterior da valva tricúspide foi retirada, inteiramente, com cordas e músculo papilar. Na primeira variante técnica, a cúspide anterior da valva tricúspide foi reparada e, na segunda, através, da plicatura do anel, a valva tricúspide foi transformada em bicúspide. A peça retirada é transplantada para a valva mitral, sendo suturada a cúspide doadora com a cúspide anterior da valva mitral e o músculo papilar doador com o topo do músculo papilar posterior da valva mitral em 3 casos e com o músculo papilar anterior e 1 caso. Os 4 pacientes foram reestudados clínica e laboratorialmente aos 15,11,2 e 1 mês de pós-operatório, respectivamente, com execelente evoluçāo. Apesar da limitada experiência, acreditamos ser esta nova técnica uma boa alternativa para o tratamento cirúrgico da insuficiência mitral por rotura de cordas tendíneas da cúspide anterior.

DESCRITORES: cordas tendíneas, autotransplante; cordas tendíneas, rotura; valva mitral, cirurgia; valva mitral, insuficiência.

\section{INTRODUÇĀO}

A insuficiência valvar mitral por rotura das cordas tendíneas da cúspide anterior tem sido tratada, cirurgicamente, através de métodos conservadores, desde o início da cirurgia cardíaca com o auxílio da circulaçāo extracorpórea, tendo sido a anuloplastia a técnica mais utilizada ${ }^{6.8}$. McGOON ${ }^{7}$, em 1959, propôs a plicatura da cúspide anterior com as cordas rotas para a correçāo do prolapso valvar mitral. CARPENTIER et alii ', 1978, introduziram a técnica de transferência de cordas tendíneas da cúspide posterior para a anterior, método efetivo que evita a ressecçāo da cúspide anterior da valva mitral e, ao mesmo tempo, preserva sua mobilidade. A criação de uma nova corda a partir de um retalho de tecido da cúspide anterior também tem sido utilizada para correção do prolapso nos casos de rotura de cordas da cúspide anterior ${ }^{5}$. Alguns achados anatômicos podem impedir o emprego das técnicas acima descritas, especialmente cordas rotas ou muito

Trabalho realizado no Departamento de Clínica Cirúrgica da Faculdade de Medicina da Fundaçảo Universidade Estadual de Londrina e no Hospital Evangélico de Londrina, Londrina, PR. Brasil.

Apresentado ao $19^{\circ}$ Congresso Nacional de Cirurgia Cardíaca, São Paulo, SP, 7 a 9 de maio, 1992.

* Da Faculdade de Medicina da Fundação Universidade Estadual de Londrina e do Hospital Evangélico de Londrina.

* Do Hospital Evangélico de Londrina.

Endereço para separatas: Francisco Gregori Jr. Rua Paes leme, $1264 \mathrm{Apt}^{\circ} 701,86010$ Londrina, PR, Brasil. 
GREGORI Jr., F.; SILVA, S. S.; FAÇANHA, L. A.; MOURE, O.; GOULART, M. P.; CORDEIRO, C.; RADE, W. - Autotransplante de cordas tendíneas: nova técnica para o tratamento cirúrgico da insuficiência mitral por rotura de cordas tendíneas da cúspide anterior. Rev. Bras. Cir. Cardiovasc., 7(4):250-255, 1992.

delgadas, na cúspide posterior, impossibilitando a transferência de cordas, para a cúspide anterior. Materiais estranhos, como tecidos biológicos ${ }^{3}$ ou o politetrafluoretileno ${ }^{2}$, têm sido utilizados nestes casos, para a substituiçāo de cordas tendíneas.

Um novo procedimento alternativo para o tratamento cirúrgico reparador do prolapso valvar por rotura de cordas tendíneas da cúspide anterior é proposto. Este consiste no autotransplante de cordas da valva tricúspide para a valva mitral, evitando-se, assim, a troca valvar ou o emprego de materiais estranhos para substituiçāo de cordas tendíneas.

Sāo apresentados os aspectos técnicos das duas variantes utilizadas, assim como a evolução pósoperatória clínica e laboratorial de 4 pacientes submetidos a este tipo de tratamento cirúrgico, com sucesso.

\section{Relato dos Casos e Técnicas Cirúrgicas}

Caso 1 - ㄴ. A. C., paciente feminina de 38 anos foi admitida no hospital em classe funcional IV (NYHA) com sopro sistólico grau $5 / 6$ em área mitral.

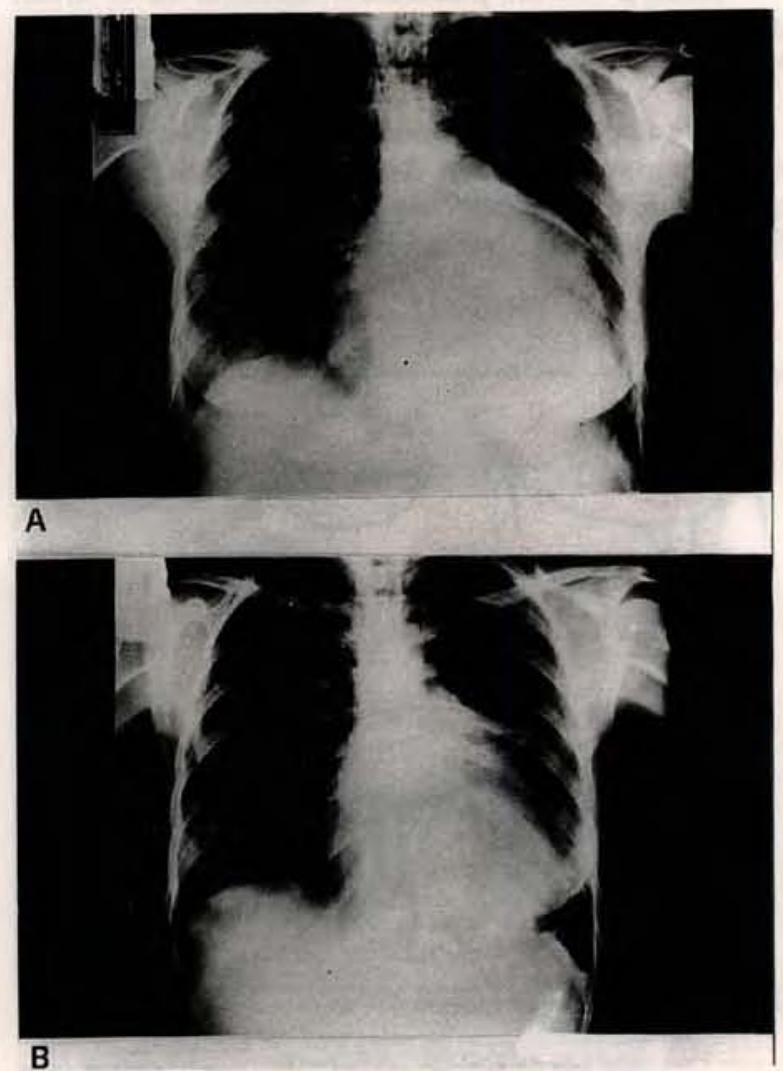

Fig. 1 - Radiografia de tórax (Caso 1)

A - Pré-operatório. Importante aumento da área cardíaca e abaulamento dos arcos do tronco pulmonar e do átrio esquerdo. B-Pós-operatório. Área cardíaca significativamente reduzida.
A radiografia de tórax mostrava importante cardiomegalia devido a aumento de câmaras esquerdas e abaulamento do tronco pulmonar (Figura 1A). A cineventrículografia esquerda demonstrava regurgitaçāo valvar mitral importante (Figura 2).

A paciente foi submetida a cirurgia cardíaca com auxílio da circulaçăo extracorpórea e desclampeamento intermitente da aorta. O átrio esquerdo foi aberto e o aspecto macroscópico da valva mitral sugeria, fortemente, tratar-se de etiologia reumática. Havia dilatação ântero-posterior do anel mitral, mais acentuado à direita, cúspides intactas e rotura de $2 / 3$ de cordas tendíneas da cúspide anterior, correspondentes ao músculo papilar posterior da valva mitral. Em seguida, o átrio direito foi aberto e a valva tricúspide foi analisada, detalhadamente. A cúspide posterior apresentava cordas tendíneas e músculo papilar muito delgados. Por outro lado, a cúspide anterior apresentava cordas com boa textura e músculo papilar bem desenvolvido. Através de ressecção retangular foi destacado um retalho da cúspide anterior, que foi liberado com suas cordas

A
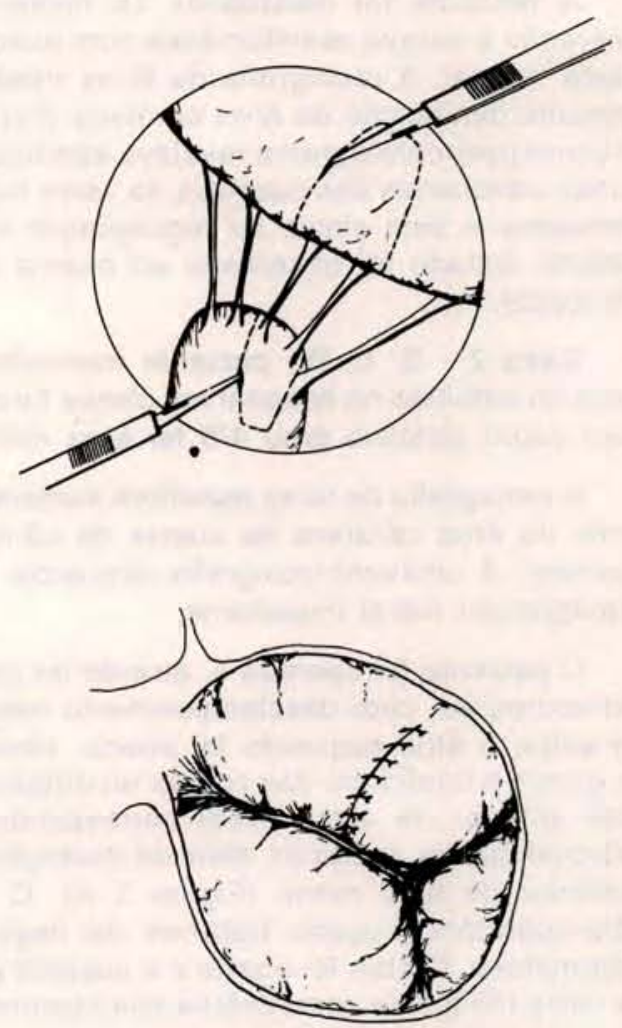

B

Fig. 2 - Desenho esquemático da cúspide anterior da valva tricúspide. A. Um fragmento da cúspide do músculo papilar com cordas integras é retirado.

B - A valva tricúspide é reparada com pontos separados de Polipropileno 5-0. 
GREGORI Jr., F.; SILVA, S. S.; FAÇANHA, L. A.; MOURE, O.; GOULART, M. P.; CORDEIRO, C.; RADE, W. - Autotransplante de cordas tendíneas: nova técnica para o tratamento cirúrgico da insuficiência mitral por rotura de cordas tendíneas da cúspide anterior. Rev. Bras. Cir. Cardiovasc., 7(4):250-255, 1992.

tendíneas correspondentes. Após secçāo longitudinal do músculo papilar, uma peça foi liberada, contendo cordas tendíneas do músculo papilar. As duas bordas da cúspide anterior da valva tricúspide foram aproximadas com pontos separados de Polipropileno 5 0 , deixando-se, assim, a valva tricúspide competente (Figura 2 B). A seguir, a peça retirada foi transplantada para a cúspide da valva mitral suturandose o músculo papilar doador ao topo do músculo papilar, posterior da valva mitral com fios de Polipropileno 5-0 ancorados em pequenos retalhos de Dacron. Com o mesmo tipo de fio, a cúspide doadora foi suturada na face ventricular da cúspide anterior da valva mitral, próxima à borda de inserção das cordas rotas. Para corrigir a dilatação anular mitral, um anel protético aberto ${ }^{4}$ foi implantado, ficando a valva mitral competente ao teste com o coração batendo. O tempo de circulaçāo extracorpórea foi de 67 minutos e o de anoxia do miocárdio de 33 minutos. A paciente evoluiu, satisfatoriamente, e recebeu alta hospitalar 10 dias após a operaçāo, quando a cineventrículografia esquerda mostrava ausência de regurgitação valvar mitral e a cineventrículografia direita regurgitação tricúspide discreta.

A paciente foi reestudada 15 meses após a operação e estava assintomática com ausculta cardíaca normal. A radiografia de tórax mostrava importante diminuição da área cardíaca (Figura 1 B). O ecodopplercardiograma revelava abertura e mobilidade adequadas das cúspides da valva mitral, sem prolapsos e sem sinais de regurgitação valvar. $O$ mesmo achado foi observado ao exame da valva tricúspide.

Caso 2 - G. L. R., paciente masculino de 27 anos foi admitido no hospital em classe funcional III, com sopro sistólico grau $4 / 6$ na área mitral.

A radiografia de tórax mostrava aumento importante da área cardíaca às custas de câmeras esquerdas. A cineventriculografia esquerda revelava regurgitaçäo mitral importante.

O paciente foi operado e, através de circulação extracorpórea, com desclampeamento intermitente da aorta, o átrio esquerdo foi aberto. Havia rotura de quase a totalidade das cordas tendíneas da cúspide anterior da valva mitral correspondentes ao músculo papilar posterior, além de dilatação ânteroposterior do anel mitral (Figura $3 \mathrm{~A}$ ). $\mathrm{O}$ aspecto intra-operatório sugeria tratar-se de degeneraçăo mixomatosa. O átrio foi aberto e a cúspide posterior da valva tricúspide apresentava boa sustentaçāo de cordas tendíneas e músculo papilar de bom tamanho. A peça foi ressecada em bloco contendo cúspide, cordas e músculo papilar. Através de plicatura do anel, a valva tricúspide foi transformada em bicúspide (Figura $3 \mathrm{~B}$ ), ficando a valva competente ao teste com injeção de solução salina no ventrículo direito.
A peça retirada foi transplantada para a valva mitral. Inicialmente, foi suturado o músculo papilar doador com o músculo papilar posterior da valva mitral e, em seguida, a cúspide doadora com a cúspide anterior da valva mitral com cordas rotas (Figura 3 C). Estas suturas foram realizadas com fios de Polipropileno 5-0 em pontos separados. Após o transplante, foi implantado anel aberto ${ }^{4}$ para a correção da dilatação do anel mitral. A valva mitral mostrouse competente ao teste com o coraçāo batendo. Os tempos de circulaçăo extracorpórea e anoxia do miocárdio foram de 107 a 75 minutos, respectivamente.

O paciente recebeu alta em boas condiçōes hemodinâmicas aos 10 dias de pós-operatório, quando a cineventrículografia esquerda mostrava valva mitral competente e valva tricúspide com discreta regurgitação. O paciente foi revisto aos 11 meses de pós-operatório, estando assintomático, sem sopros. A radiografia de tórax mostrava significativa diminuiçāo de área cardíaca. $O$ ecodopplercardiograma mostrava ausência de estenose e prolapso mitral, boa mobilidade das cúspides sem regurgitaçāo mitral e tricúspide (Figura 4).

Caso 3 - A. M., paciente masculino de 67 anos, classe funcional IV e sopro sistólico $6 / 6$ à admissāo hospitalar.

A radiografia de tórax mostrava aumento moderado da área cardíaca às custas de câmaras esquerdas e a cineventriculografia esquerda demonstrava regurgitação mitral importante (Figura $5 \mathrm{~A}$ ).

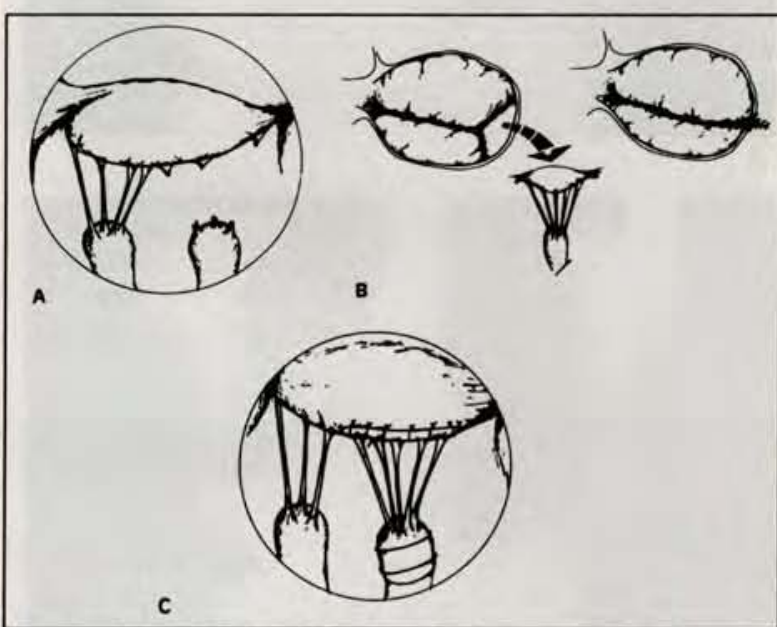

Fig. 3 - Desenho esquemático (Caso 2).

A - Cúspide anterior da valva com cordas rotas.

B - Retirada da cúspide posterior da valva tricúspide a ser transplantada para valva mitral com transformaçáo da valva tricúspide em bicúspide.

C - Cúspide anterior da valva mitral após o transplante de cordas tendíneas da valva tricúspide. 
GREGORI Jr., F.; SILVA, S. S.; FAÇANHA, L. A.; MOURE, O.; GOULART, M. P.; CORDEIRO, C.; RADE, W. - Autotransplante de cordas tendíneas: nova técnica para o tratamento cirúrgico da insuficiência mitral por rotura de cordas tendíneas da cúspide anterior. Rev. Bras. Cir. Cardiovasc., 7(4):250-255, 1992.
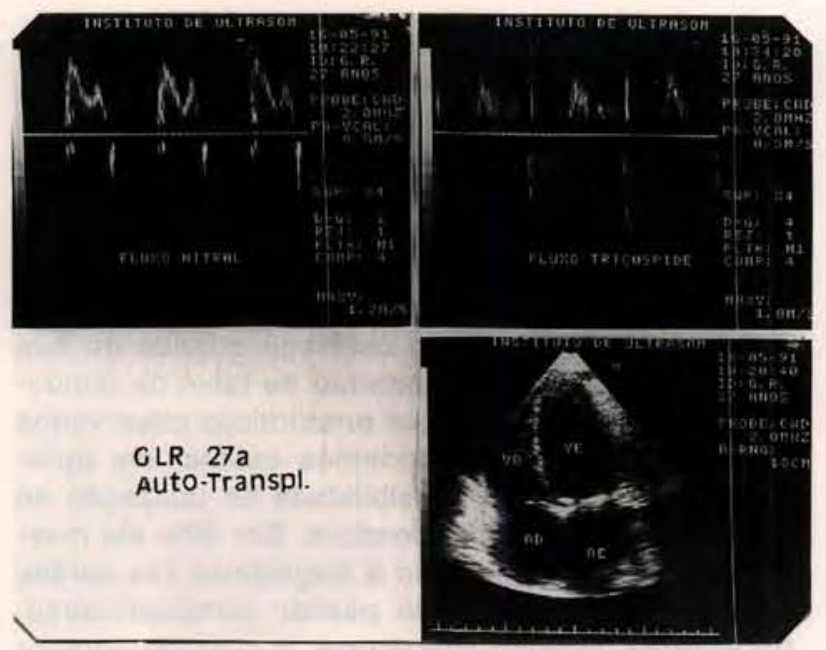

Fig. 4 - Ecodopplercardiograma pós-operatório do paciente submetido ao transplante de cordas tendíneas utilizando-se a cúspide posterior da valva tricúspide. Note boa coaptação das cúspides da valva mitral (inferior) a ausência de refluxo mitral e tricúspide (superior).

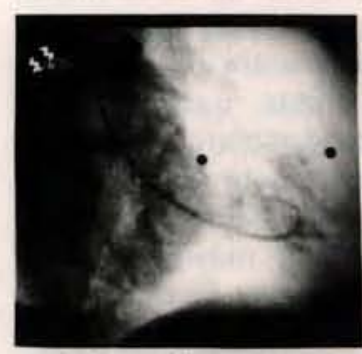

$\dot{\mathbf{A}}$

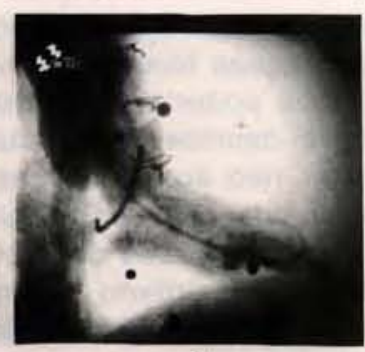

B

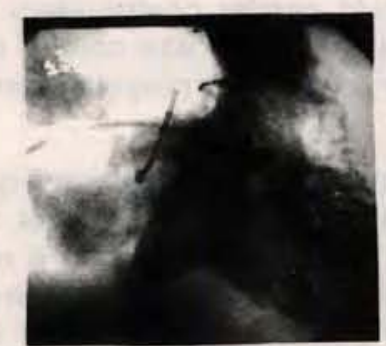

C

Fig. 5 - Aspectos cineventrículográficos (Caso 3).

A - Pré-operatório. Regurgitação mitral importante.

B - Pós-operatório. Discreta regurgitaçảo mitral.

C - Pós-operatório. Discreta regurgitação tricúspide.

A cirurgia foi realizada com auxílio de circulação extracorpórea e desclampeamento intermitente da aorta. O átrio esquerdo foi aberto e os achados sugeriram tratar-se de degeneração mixomatosa. Havia dilatação do anel mitral, prolapso da cúspide anterior por alongamento e por rotura de cordas tendíneas, o mesmo acontecendo com a cúspide posterior. Inicialmente, foi realizada ressecção retangular da cúspide posterior da valva mitral para correçāo do prolapso devido a rotura das cordas neste local e, em seguida, foi realizado encurtamento de cordas em nível supravalvar na cúspide anterior próximo à comissura ântero-lateral. Para correção do prolapso da cúspide anterior da valva mitral causada pela rotura de cordas foi realizado um autotransplante de cordas da valva tricúspide para a valva mitral. Foi retirada a cúspide posterior da valva tricúspide, transformando-a, assim, em bicúspide. Esta cúspide apresentava cordas tendíneas com boa textura e músculo papilar bem dimensionado que foi suturado ao topo do músculo posterior da valva com fios de Polipropileno 5-0. A seguir, após divisão longitudinal da cúspide doadora, parte desta foi suturada à cúspide anterior da valva mitral e parte à cúspide posterior, corrigindo-se, assim, o prolapso da cúspide anterior por rotura de cordas e o prolapso da cúspide posterior por alongamento de cordas (Figura 6). A mesma peça, portanto, forneceu cordas tendíneas para a cúspide anterior e para a cúspide posterior da valva mitral. Um anel protético aberto ${ }^{4}$ foi implantado para correção da dilatação do anel mitral, ficando a valva competente ao teste intra-operatório. Os tempos de circulação extracorpórea e anoxia do miocárdio foram 99 e 69 minutos, respectivamente.

O paciente recebeu alta hospitalar em boas condiçōes 11 dias após a operação, quando a cineventrículografia mostrava regurgitação mitral e tricúspide discretas (Figuras $5 \mathrm{~B}$ e $5 \mathrm{C}$ ).

Dois meses após a operação, o paciente encontrava-se assintomático, com sopro sistólico de 2/6 em área mitral. A radiografia de tórax mostrava reduçāo de área cardíaca e o ecodopplercardiograma mostrava ausência de estenose e prolapso mitral, discreta regurgitação mitral e valva tricúspide competente.

Caso 4 - G. L. F. B. - Paciente feminina de 63 anos, classe funcional III e com sopro sistólico em área mitral $5 / 6$ foi admitida no hospital.

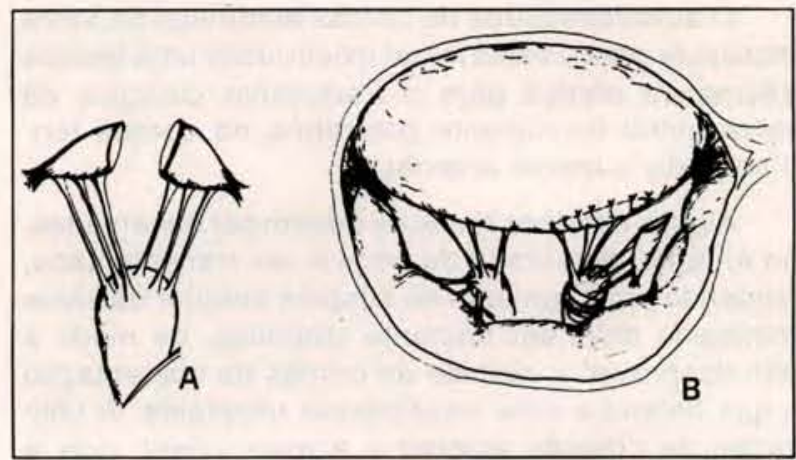

Fig. 6 - Desenho esquemático (Caso 3)

Cúspide posterior da valva tricúspide pronta para ser transplantada. (A). - A secçáo longitudinal da cúspide permite a fixaçáo de uma das partes da cúspide anterior da valva mitral e a outra parte da cúspide posterior da valva mitral (B). 
GREGORI Jr., F.; SILVA, S. S.; FAÇANHA, L. A.; MOURE, O.; GOULART, M. P.; CORDEIRO, C.; RADE, W. - Autotransplante de cordas tendíneas: nova técnica para o tratamento cirúrgico da insuficiência mitral por rotura de cordas tendíneas da cúspide anterior. Rev. Bras. Cir. Cardiovasc., 7(4):250-255, 1992.

A radiografia de tórax apresentava aumento importante de área cardíaca às custas de câmeras esquerdas. A cineventrículografia esquerda revelava refluxo importante de contraste do ventrículo esquerdo para o átrio esquerdo.

A cirurgia foi realizada com auxílio da circulaçāo extracorpórea e desclampleamento intermitente da aorta. Após a abertura do átrio esquerdo, foi observada valva mitral com aspecto de degeneraçāo mixomatosa. $O$ anel mitral era dilatado posteriormente e havia rotura de cordas tendíneas da cúspide anterior relativa ao músculo papilar anterior da valva mitral. Em seguida, o átrio direito foi aberto e a cúspide posterior da valva mitral era inapropriada para o autotransplante. Foi retirada uma peça da cúspide anterior da valva tricúspide, através de ressecção retangular $e$, em seguida, foi transplantada a valva mitral segundo a técnica descrita no primeiro caso. Desta feita, o músculo papilar da peça doadora foi suturado ao topo do músculo papilar anterior. Um anel aberto foi implantado para correçāo da dilatação do anel mitral e a valva ficou competente ao teste intra-operatório. O tempo de circulação extracorpórea foi de 87 minutos e o de anoxia do miocárdio de 58 minutos.

A paciente evoluiu assintomática e sem sopros, recebendo alta hospitalar no oitavo dia de pós-operatório. A radiografia de tórax já revelava diminuiçāo da área cardíaca e o estudo hemodinâmico mostrava valva mitral competente (Figura 13 - inferior A) e valva tricúspide com regurgitaçāo discreta.

O ecodopplercardiograma mostrava boa abertura e mobilidade das cúspides, ausência de prolapso mitral e tricúspide, sem regurgitação mitral e insuficiência tricúspide discreta.

\section{COMENTÁRIOS}

O autotransplante de cordas tendíneas da valva tricúspide para a valva mitral mostrou ser uma técnica alternativa efetiva para o tratamento cirúrgico da valva mitral insuficiente por rotura de cordas tendíneas da cúspide anterior.

Alguns detalhes técnicos devem ser observados, no entanto. A retirada da peça a ser transplantada, contendo um fragmento da cúspide anterior da valva tricúspide deve ser bastante criteriosa, de modo a nāo desprover a cúspide de cordas de sustentação - que levaria a uma insuficiência tricúspide. A utilizaçāo da cúspide anterior é a mais viável, pois é aquela que apresenta maior número de cordas e músculos papilares de dimensōes adequadas, o que facilita sua sutura na ocasiāo de sua fixação no músculo papilar da valva mitral.
A alternativa, ao nosso ver, preferencial, quando possível, é a retirada completa da cúspide posterior, transformando, assim, a valva tricúspide em bicúspide. Este procedimento nāo torna a valva estenótica, uma vez que o anel da valva tricúspide é normal ou, em geral, aumentado nos pacientes portadores de lesōes da valva mitral. A abordagem da cúspide septal năo achamos aconselhável, podendo surgir distúrbios do ritmo cardíaco quando da sua reconstruçāo por tracionamento do feixe de conduçāo. Baseados em estudos anatômicos observados em coraçōes normais, podemos estimar em aproximadamente $70 \%$ a possibilidade de utilizaçăo da cúspide posterior como doadora. Em $30 \%$ ela mostra-se inapropriada devido à fragilidade das cordas tendíneas ou do músculo papilar correspondente. No entanto, quando isto ocorre, a cúspide anterior é de tamanho razoável, assim como o número de cordas tendíneas e tamanho do músculo papilar.

As suturas, tanto da reconstruçāo da valva tricúspide como da fixação do retalho da cúspide na face ventrícular da cúspide anterior da valva mitral, já sāo bem conhecidas e, em geral, não apresentam complicaçōes tardias. A sutura entre os músculos papilares poderia ser questionada, quanto a uma possível necrose do músculo transplantado. Desde o início, nāo acreditávamos que este fato pudesse ocorrer, pois este tipo de sutura já é de longo tempo utilizado nos casos de ruptura de músculo papilar secundária a infarto do miocárdio. O tratamento cirúrgico da insuficiência mitral pode ser conservador, nestes casos, quando o músculo papilar roto é suturado ao músculo papilar contra-lateral mantendose, assim, a sustentaçăo das cordas tendíneas e corrigindo-se o prolapso da cúspide anterior da valva mitral.

A nova técnica apresentada tem a qualidade de proporcionar as condiçōes fisiológicas de abertura e fechamento da valva mitral, sem a necessidade de emprego de materiais estranhos com o pericárdio bovino ${ }^{3}$ ou o Politetrafloretileno ${ }^{2}$ para substituição das cordas tendíneas.

O autotransplante de cordas, utilizado nos casos de rotura de cordas tendíneas da cúspide anterior da valva mitral pode estender-se para os casos de estenose mitral com calcificaçāo localizada da cúspide anterior com comprometimento das cordas tendíneas. Neste caso, nāo somente as cordas tendíneas seriam substituídas, como também parte da cúspide anterior, evitando-se, assim, uma troca valvar.

Os excelentes resultados observados autorizam o emprego do autotransplante de cordas, procedimento como técnica alternativa para o tratamento cirúrgico reparador da insuficiência mitral por rotura de cordas tendíneas da cúspide anterior. 
GREGORI Jr., F.; SILVA, S. S.; FAÇANHA, L. A.; MOURE, O.; GOULART, M. P.; CORDEIRO, C.; RADE, W. - Autotransplante de cordas tendíneas: nova técnica para o tratamento cirúrgico da insuficiência mitral por rotura de cordas tendíneas da cúspide anterior. Rev. Bras. Cir. Cardiovasc., 7(4):250-255, 1992.

\section{RBCCV 44205-186}

GREGORI Jr.,F.; SILVA, S. S.; FAÇANHA, L. A.; MOURE, O.; GOULART, M. P.; CORDEIRO, C.; RADE, W. -Autotransplantation of chordae tendineae: new technique for the surgical approach of mitral insufficiency. Rev. Bras. Cir. Cardiovasc., 7(4):250-255, 1992.

ABSTRACT: One of the most frequent causes of mitral valve prolapse is rupture of the chordae tendineae. Several techniques have been used to correct severe mitral regurgitation. However, mitral valve replacement is the principal surgical approach to it. We describe a technique used in four patients with marked mitral regurgitation due to ruptured chordae tendineae of the anterior leaflet. We have grafted chordae tendineae from the tricuspid valve onto the mitral valve. In the first and fourth case we removed part of the anterior leaflet of the tricuspid valve, together with chordae tendineae and part of the papillary muscle. In the other two cases we removed the posterior leaflet, together with its chordae tendinease and papillarly muscle. In the first and fourth cases the tricuspid valve was repaired. In the other two cases it was turned into bicuspid valve by means of an annular plication. The first extremity of the graft to be sutured was the papillary muscle, followed by the leaflet. Studies carried out 15, 11, 2 and 1 month, postoperatively, showed that both valves, i. e., mitral and tricuspid, were competent and the patients were asymptomatic. In spite of our limited experience, we believe, this technique is a sensible alternative to mitral valve replacement.

DESCRIPTORS: cordae tendineae, autotransplantation; cordae tendineae, rupture; mitral valve, surgery; mitral insufficiency.

\section{REFERÊNCIAS BIBLIOGRÁFICAS}

1

CARPENTIER, A.; RELLAND, J.; DELOCHE, A.; PIWINICA, A.; CHAUVAUD, S.; DUBOST, C. - Conservative management of the prolapsed mitral valve. Ann. Thorac. Surg., 26: 294-301, 1978.

DAVID, T. E. - Replacement of chordae tendineae with expanded polytetrafluorethylene sutures. J. Cardiac. Surg., 4: 286-290, 1989.

FRATER, R. W. M.; GABBAY, S.; SHORE, D.; FACTOR, S.; STROM, J. - Reproducible replacement of elongated or ruptured mitral valve chordae. Ann. Thorac. Surg., 35: 14-19, 1983.

GREGORI Jr., F.; SILVA, S. S.; BABA, K.; QUEIROZ, L.; TAKEDA, R.; FAÇANHA, L.; HAYASHI, S.; CANESIN, O. - Um novo modelo de anel protético para acidentes com insuficiência valvar mitral: relato de dois casos. Arq. Bras. Cardiol., 50: 417-420, 1988.
5 GREGORI Jr., F.; TAKEDA, R.; SILVA, S. S.; FAÇANHA, L.; MEIER, M. A. - A new technique for repair of mitral insufficiency caused by ruptured chordae of the anterior leaflet. J. Thorac. Cardiovasc. Surg., 96: $765-368,1988$.

6 LILLEHEI, C. W.; GOTT, V. L.; De WALL, R. A.; VASCO, R. L. - Surgical correction of pure mitral insufficiency by annuloplasty under direct vision. Lancet, 11: 446$451,1952$.

7 McGOON, D. C. - Repair of mitral insufficiency due to ruptured chordae tendineae. J. Thorac. Cardiovasc. Surg., 30: 357-362, 1960.

8 MERENDINO, K. A.; THOMAZ, G. I.; JESSEH, J. E.; HERON, P. W.; WINTERSHELD, L. C.; VETO, R. R. - The open correction of mitral regurgitation and or stenosis with special reference to regurgitation treated by medid annuloplasty utilizing a pump oxygenator. Ann. Surg., 150: 5-15, 1959. 